\title{
Dynamics of Jupiter's Cloud Bands ${ }^{1}$
}

\author{
Andrew P. Ingersoll and JefFrey N. Cuzzi \\ Division of Geological Sciences, California Institute of Technology, Pasadena
}

(Manuscript received 15 May 1969)

\begin{abstract}
The observed zonal motion of Jupiter's atmosphere near the cloud tops is investigated assuming geostrophic balance and a systematic temperature difference between light and dark bands. Excellent agreement is obtained between observed velocities and those predicted from the thermal wind relation with the temperature and rotation rate of the deep atmosphere independent of latitude. The light bands are found to be warmer than the dark bands. This model is inconsistent with a monotonic variation of insolation as the only energy source for the flow.

The barotropic stability criterion is also applied to the observed motion, and it appears that the necessary criterion for instability is approached but not exceeded in mid-latitude regions.
\end{abstract}

\section{Tatroduction}

Peek (1958) has given a detailed account of cloud motions on Jupiter observed over the last 50 years. The velocity is almost entirely zonal, and this zonal velocity varies with latitude such that the observed rotation periods of cloud markings range from $9^{\mathrm{h}} 49^{\mathrm{m}}$ to almost $9^{\mathrm{h}} 59^{\mathrm{m}}$. The shorter rotation periods are characteristic of the equatorial region, but at mid-latitudes both extremes may co-exist in adjacent bands separated by less than $10^{\circ}$ of latitude. At any given time, the distribution of rotation rates with latitude appears to be correlated with the visual appearance of the planet. Thus, Peek gives the rotation periods of some 20 recognized currents, together with the locations of these currents in relation to nearby belts (dark bands) and zones (light bands). He also specifies the approximate latitude at which each current is most frequently observed. These observations are described in the first half of Peek's book, and are summarized in a convenient table on pp. 189-190.

We propose to utilize these data in order to test the hypothesis of geostrophic balance as it applies to Jupiter, assuming a systematic temperature difference between light and dark bands. We shall also investigate the relevance of the barotropic stability criterion using these data, assuming a smooth variation of zonal velocity between Peek's individual datum points.

The basic hypothesis made in testing the geostrophic approximation on Jupiter is that the differential rotations are associated with horizontal temperature differences between belts and zones, and that these differences vanish at depth in the atmosphere. The deep atmosphere is assumed to be in solid rotation, with rota-

\footnotetext{
${ }^{1}$ Contribution No. 1637 of the Division of Geological Sciences,
} California Institute of Technology, Pasadena. tion period $9^{\mathrm{h}} 55^{\mathrm{m}} 30^{\mathrm{s}}$. This is the period of Jupiter's magnetic field (Warwick, 1967), and it is also typical of the visible atmosphere at mid-latitudes. Solid rotation of the deep atmosphere implies that the fluid is capable of transmitting shearing stresses; nevertheless, we treat the fluid as inviscid in the vicinity of the cloud tops. This assumption is self-consistent for a fluid of small viscosity; shearing stresses will make themselves felt in the lower atmosphere by virtue of the great depth of that region compared to the region near the cloud tops. The same results apply if there is a solid surface at intermediate depth capable of transmitting shearing stress.

The geostrophic approximation leads to a thermal wind equation, relating the vertical shear in the zonal velocity to the latitudinal gradient of temperature. This equation is integrated upward from the deep interior to the visible cloud surface, and in this way the form of the function relating zonal velocity and latitude is obtained theoretically from the visible band structure. This function is proportional to the unknown temperature difference between light and dark bands, and to the unknown depth in the atmosphere at which these differences vanish.

The barotropic stability criterion is a condition on the sign of the gradient of absolute vorticity, $-U^{\prime}+2 \Omega_{0}$ $\sin \Phi$, where $U=U(y)$ is the zonal velocity profile, $y$ the poleward coordinate, $\Omega_{0}$ a rotation rate characteristic of the planet, and $\Phi$ the latitude. According to this criterion, a necessary condition for instability is that this gradient change sign. Thus, the vorticity gradient $U^{\prime \prime}$ must be compared with the quantity, $\beta=\left(2 \Omega_{0} / a\right)$ $\cos \Phi$, where $a$ is the radius of the planet. The quantity $U^{\prime \prime}$ will be greatest in regions of minimum $U$ (retrograde flow relative to that in adjacent regions). If $U^{\prime \prime}$ approaches, but does not exceed, $\beta$ in these regions, the 
mechanism of barotropic instability is probably important in limiting the amplitude of the motion. If $U^{\prime \prime} / \beta \ll 1$ everywhere, then other mechanisms must be limiting the amplitude. If $U^{\prime \prime} / \beta>1$, then some additional stabilizing mechanism must exist.

The theoretical analysis is extremely simple, and the analysis of Peek's data is straightforward. The validity of the assumptions is verified by the excellent agreement between predicted and observed zonal velocities, and by the fact that the necessary criterion for barotropic instability is approached but not exceeded at mid-latitudes. These results provide a powerful constraint on theories of Jupiter's cloud motions. With further data on the albedos of the belts and zones, on their temperatures, pressures and composition, we should be able to understand the basic dynamical mechanisms at work in Jupiter's atmosphere.

\section{Analysis}

A casual reading of the aforementioned table of Peek's book reveals that the shortest rotation periods are associated with the equatorward edges of belts (poleward edges of zones). Similarly, the longest rotation periods are associated with the poleward edges of belts (equatorward edges of zones). Intermediate rotation periods are associated with the middle of belts and the middle of zones. While this is consistent with the thermal wind relation with zones hotter than belts, the agreement is more striking when the proper geometric factors, which depend on latitude, are taken into account.

Assume steady, zonal motion of an inviscid ideal gas. Let $\Phi$ be latitude, positive in the Northern Hemisphere, and let $U$ be velocity to the east, relative to a reference frame rotating with constant angular velocity $\Omega_{0}$. A crucial assumption of the analysis is that the deep atmosphere below the clouds is in solid rotation with this angular velocity. We choose $\Omega_{0}$ from the radio rotation period, $9^{\mathrm{h}} 55^{\mathrm{m}} 30^{\mathrm{s}}$.

Let $g$ be the magnitude of the resultant of the gravitational acceleration and the centrifugal acceleration associated with the angular velocity $\Omega_{0}$. Let $z$ be a coordinate in the direction opposite this vector. Since the vertical thickness of the region of interest is small relative to the radius of the planet $a$, and since the centrifugal acceleration is small compared to the gravitational acceleration, we shall assume that the local vertical coincides with the radius vector from the center of the planet, and we shall treat $g$ as a constant. Finally, since the vertical scale of motion is much less than the horizontal scale, we shall neglect vertical accelerations, and shall assume that the fluid is in strict hydrostatic equilibrium. These are a standard set of assumptions, well known in geophysical fluid dynamics (Phillips, 1963).

We assume that the motion of Jupiter's atmosphere is steady, zonal and axially symmetric. Then the equations of motion can be written

$$
\begin{aligned}
2 \Omega_{0} U \sin \Phi & =-\frac{1}{\rho a} \frac{\partial P}{\partial \Phi}, \\
g & =-\frac{1}{\rho} \frac{\partial P}{\partial z}
\end{aligned}
$$

where $P$ is the pressure and $\rho$ the density of the fluid. Letting $T$ be temperature we have the ideal gas law

$$
P=\rho R T,
$$

where $R$ is the gas constant of Jupiter's atmosphere. From the above, it is not difficult to derive the thermal wind relation,

$$
2 \Omega_{0} \sin \Phi\left(\frac{\partial U}{\partial z}\right)_{\Phi}=-\frac{g}{T a}\left(\frac{\partial T}{\partial \Phi}\right)_{P}
$$

This equation may be integrated to give

$$
2 \Omega_{0} U(z, \Phi) \sin \Phi=-\frac{g}{a} \int_{-\infty}^{z} \frac{1}{T}\left(\frac{\partial T}{\partial \Phi}\right)_{P} d z
$$

since $U$ is measured relative to the uniform rotation of the deep atmosphere.

Our aim is to test the validity of (5), assuming a systematic temperature difference between belts and zones. The left side may be evaluated directly from the rotation periods given by Peek, using the relation

$$
U(z, \Phi)=a \cos \Phi\left[\Omega(z, \Phi)-\Omega_{0}\right]
$$

where $z$ is now the height of the visible cloud surface. The right side may be evaluated, under the assumptions stated, from the visible band structure given by Peek. Let $\Delta T$ be the typical difference in temperature between a zone and a belt ( $\Delta T>0$ implies zones hotter). Let $\Delta z$ be the typical vertical distance between the cloud top and the level at which the atmosphere is in solid rotation. Then an estimate of the right side of (5) is

$$
-\frac{g}{a} \int_{-\infty}^{z} \frac{1}{T}\left(\frac{\partial T}{\partial \Phi}\right)_{P} d z=-\frac{g}{a}\left(\frac{m \Delta T}{T \Delta \Phi}\right) \Delta z,
$$

where $m=0, \pm 1$ as follows:

$$
m=\left\{\begin{aligned}
+1 & \text { ( } \mathrm{N} \text { edge of belt, } \mathrm{S} \text { edge of zone) } \\
0 & \text { (middle of belt or zone) } \\
-1 & \text { (S edge of belt, } \mathrm{N} \text { edge of zone })
\end{aligned}\right.
$$

Here $g, \Delta T, \Delta z$ and $T$ are all constants, and $a \Delta \Phi$ is the width of the current, taken to be one-half the distance from the middle of the belt on one side of the current to the middle of the zone on the other side. Then taking $g=2650 \mathrm{~cm} \mathrm{sec}^{-2}, a=71,400 \mathrm{~km},(\Delta T / T) \Delta z=1.0 \mathrm{~km}$, we obtain the results shown in Fig. 1 . The circles are obtained from direct observation, by (6), and the crosses 


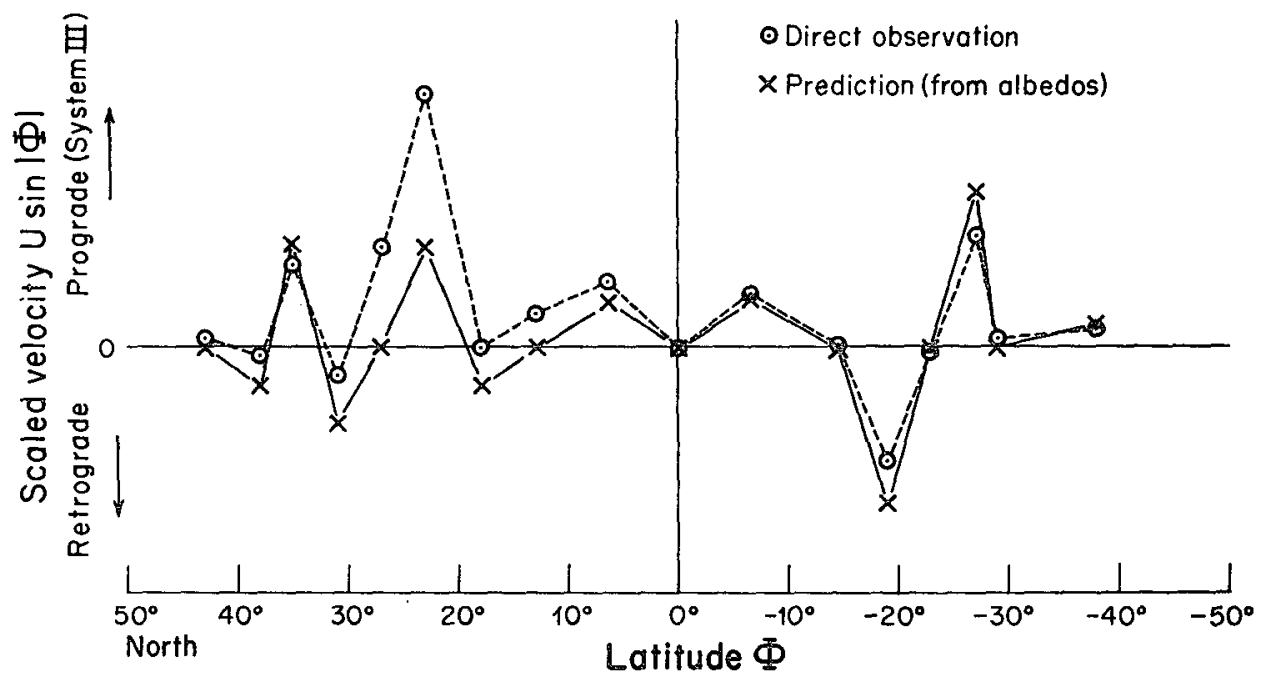

FIG. 1. Comparison of observed zonal velocity and that predicted from the visual albedo gradient. The observational data are from Peek (1958). Velocity is measured relative to System III, in arbitrary units. The factor $\sin |\Phi|$, which suppresses data at the equator, arises naturally from the geostrophic approximation. The sense of the correlation indicates that zones are hotter than belts.

from the visual band structure, by (7) and (8). The best fit between the two sets of data yields the estimate

$$
(\Delta T / T) \Delta z=1.2 \pm 0.2 \mathrm{~km} \text {. }
$$

This striking agreement between two independent sets of data demands some discussion. The only ambiguity arises in choosing a value of $m$, and this is only a problem at the extreme end points in each hemisphere and at the south edge of the South Equatorial Belt. Peck gives two rotation periods for this current : the first nearly coincides with the period for the middle of the South Equatorial Belt (p. 107); the second is more typical of the poleward edges of belts. In Fig. 1 we have treated the first entry as belonging to the middle of the belt $\left(\Phi=-14.5^{\circ}\right)$, and the second as belonging to its poleward edge $\left(\Phi=-19^{\circ}\right)$. Since the two entries refer to observations in different years, there is no real difficulty with this interpretation.

Note also that the zeros of the two curves in Fig. 1 almost coincide, indicating that the results are consistent with the deep atmosphere rotating at the radio frequency. Changing the assumed angular velocity of the deep atmosphere tends to raise or lower the circles in Fig. 1 by an additional term proportional to $\sin |\Phi|$ $\cos \Phi$. The results are consistent with any value of the period of the deep atmosphere from $9^{\mathrm{h}} 54^{\mathrm{m}}$ to $9^{\mathrm{h}} 56^{\mathrm{m}}$, although the mean period appears to be somewhat shorter in the Northern Hemisphere than in the Southern.

Before attempting to interpret these results, we shall describe another analysis of Peek's data, using the barotropic stability criterion. For zonal velocity pro-

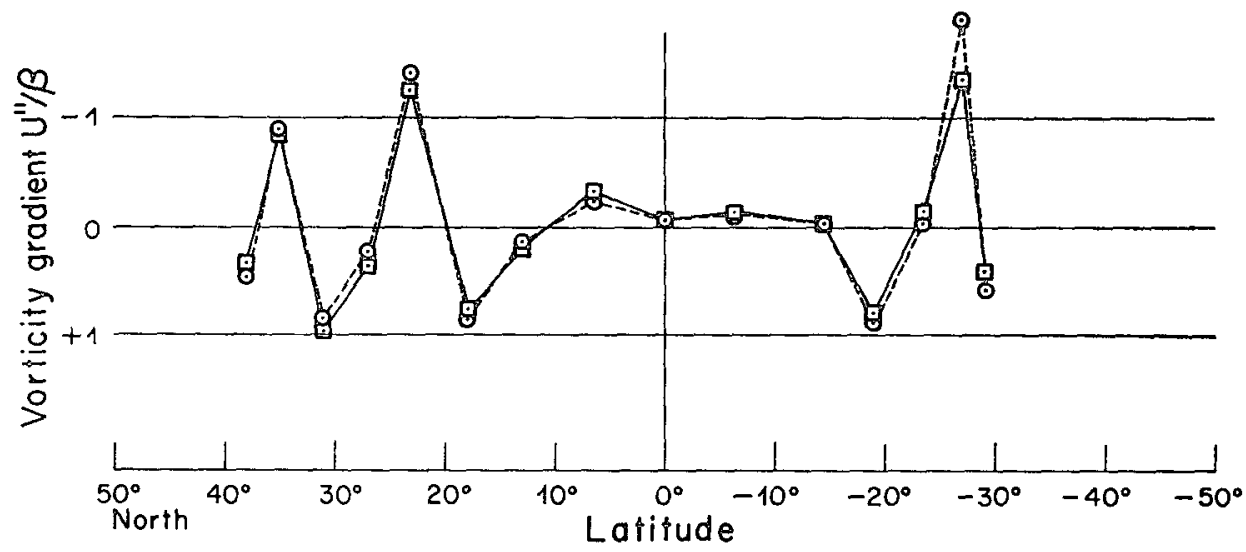

FIG. 2. Comparison of the relative vorticity gradient, $-U^{\prime \prime}$, and the planetary vorticity gradient, $\beta=\left(2 \Omega_{0} / a\right) \cos \Phi$. The second derivative $U^{\prime \prime}$ was estimated by a three-point finite difference method, using Peek's (1958) data. Squares: Peek's data treated as a point-by-point sampling of the actual flow; circles: Peek's data treated as a set of averages, band by band, of the actual flow. The barotropic stability criterion states that $U^{\prime \prime} / \beta>1$ is necessary for instability. 
files $U(y)$, where $y$ is the poleward coordinate, a necessary condition for self-excited disturbances to exist is (Kuo, 1949)

$$
U^{\prime \prime} / \beta=1, \quad \beta=\left(2 \Omega_{0} / a\right) \cos \Phi .
$$

This is simply a generalization of Rayleigh's (Lin, 1955, p. 52) criterion for the stability of parallel flows. Strictly speaking, this criterion applies only to horizontal flow on a rotating sphere in which velocity is independent of the vertical coordinate $z$. Thus, it would appear that this criterion should not apply in the present case, since we have already demonstrated that horizontal temperature differences do exist, and that the velocity $U$ depends both on $y$ and $z$, by (5). However, Pedlosky (1964) has shown that (10) is also a necessary condition for instability in a two-layer system when the velocity is not the same in the two layers. Thus, we might expect this criterion to be relevant in Jupiter's atmosphere. Moreover, since our knoweldge of the vertical structure of the zonal flow is incomplete, (10) is the only formula which can be applied to Peeks' data without further assumptions.

The second derivative $U^{\prime \prime}$ was estimated by fitting a quadratic to each set of three adjacent datum points in Peek's table. Two methods were used. In the first, each entry in Peek's data was given a single latitude and a single zonal velocity. In the second, each entry was given a latitude band, and the observed zonal velocity was treated as an average for the band. In Fig. 2 the results of the first method are indicated by squares, and the results of the second method by circles. The agreement is due mainly to the constraints imposed by three-point interpolation formulae. If the observed currents are actually narrow jets, much thinner than the resolution of Peek's data, then either method will lead to our underestimating the quantity $U^{\prime \prime} / \beta$. As in Fig. 1, only the second of Peek's entries for the south edge of the South Equatorial Belt was used; the first entry was applied to the middle of the belt.

Again we observe a striking confirmation of the validity of the model. The quantity $U^{\prime \prime} / \beta$ fluctuates between \pm 1 , but the necessary criterion (10) for instability is never satisfied. This suggests that the instability tends to limit the amplitude and wavelength of the flow such that $U^{\prime \prime}$ is always less than $\beta$.

\section{Interpretation}

A number of conclusions follow almost immediately from the observational data. First, the zonal motion is in approximate geostrophic balance, and the differential rotations are associated with horizontal temperature differences between belts and zones (strictly speaking, these are temperature differences with respect to latitude along surfaces of constant pressure). A second conclusion is that zones are hotter than belts, which follows from (9) with $\Delta z>0$. (The case $\Delta z<0$ corresponds to a rigid lid above the cloud tops, with the deep atmosphere not in solid rotation. This seems unlikely in spite of Jupiter's strong magnetic field, since stresses due to collisions between ions and neutrals in the ionosphere are unlikely to be significant compared to turbulent stresses in the deep atmosphere.) A third conclusion is that the barotropic stability criterion is relevant on Jupiter. This conclusion has two parts: 1) that the instability limits the amplitude of the motion such that $U^{\prime \prime}<\beta$, and 2) that a separate mechanism exists by which the maximum value of $U^{\prime \prime}$ compatible with the stability criterion is favored.

A number of other conclusions follow after certain reasonable assumptions are made. If the rotation rate of the deep atmosphere is close to the radio frequency, that is, intermediate between the extremes observed at the cloud tops, then from (5) there must be bands where temperature increases toward the pole. It is diffcult to imagine how this circumstance could arise if the only energy source for these motions is a monotonic decrease of insolation from equator to pole, as in the baroclinic instability models of Jupiter's atmosphere (Stone, 1967; Stone et al., 1969; Gierasch and Stone, 1968). These models imply a monotonic decrease of temperature from equator to pole, which according to (5), would only be possible if the deep atmosphere were rotating more slowly than any observed band, with a period several minutes longer than the radio period. We feel there are other possible energy sources for the flow, and so we reject the baroclinic instability model of Jupiter's atmosphere.

At least two possible mechanisms for maintaining systematic temperature differences between zones and belts do exist; an internal heat source (Low, 1966; Aumann et al., 1969), and differences in the absorption of sunlight between belts and zones. By the internal heat source hypothesis, the bands are convection cells, with warm, rising fluid in the zones, and cold, sinking fluid in the belts. Thus, the light zones might be clouds, and the dark belts might be clear regions. ${ }^{2}$ Meridional motion would be from the zones to the belts. A test of this hypothesis would be to determine the height of scattering of sunlight in the zones and belts; the zones should show scattering from greater heights in the atmosphere. To discredit this hypothesis, it would be sufficient to show that the total thermal flux is greatest over the dark belts.

An alternate hypothesis is that the zones absorb more sunlight than the belts, and are therefore hotter. This could be tested by measuring the absolute albedos of the belts and zones throughout the solar spectrum. The absorption by zones in the IR would have to be much greater than that by belts, to offset the obvious darker shade of the belts in the visible.

Finally, differences in elevation might produce the required temperature differences, If the absorption of

${ }^{2}$ Note added in proof. See Westphal (1969) for a striking confirmation of this hypothesis. 
sunlight takes place at higher elevations in belts than in zones, then the atmosphere beneath the belts would be shielded from the sun. Temperature would continue to increase to considerable depths at zones, but might stay at a relatively low value at the belts. In this way, the zones might be hotter than belts on constant pressure surfaces below the cloud tops. To discredit this hypothesis, it would be sufficient to show that zones absorb light higher in the atmosphere than do the belts.

To summarize, we feel that Peek's data are inconsistent with a monotonic decrease of insolation as the only energy source for the flow. We have suggested three mechanisms, one involving an internal heat source, and two involving differences in solar heating, which might account for the observed flow. Future observations, well within present capabilities, should be able to distinguish between these alternatives.

Acknoruledgments. It is a pleasure to thank Drs. Gierasch and Stone for valuable comments on this work. Financial support was provided by the National Aeronautics and Space Administration under Grant NGL 05002-003.

\section{REFERENCES}

Aumann, H. H., C. M. Gillespie, Jr., and F. J. Low, 1969: The internal powers and effective temperatures of Jupiter and Saturn. Astrophys. J., 157, 69.

Gierasch, P. J., and P. H. Stone, 1968: A mechanism for Jupiter's equatorial acceleration. J. Atmos. Sci., 25, 1169-1170

Kuo, H. -L., 1949: Dynamic instability of two-dimensional nondivergent flow in a barotropic atmosphere. $J$. Meteor., 6, 105-122.

Lin, C. C., 1955: The Theory of Hydrodynamic Stability. Cambridge University Press, $155 \mathrm{pp}$.

Low, F. J., 1966: Observations of Venus, Jupiter, and Saturn at $\lambda 20 \mu$. Astron. J., 71, 391.

Pedlosky, J., 1964: The stability of currents in the atmosphere and the ocean: Part II. J. Atmos. Sci., 21, 342-353.

Peek, B. M., 1958: The Planet Jupiter. London, Faber and Faber, $283 \mathrm{pp}$.

Phillips, N. A., 1963: Geostrophic motion. Rev. Geopliys., 1, 123-176.

Stone, P. H., 1967: An application of baroclinic stability theory to the dynamics of the Jovian atmosphere. J. Atmos. Sci., 24, 642-652.

—, S. Hess, R. Hadlock and P. Ray, 1969: Preliminary results of experiments with symmetric baroclinic instabilities. $J$. Almos. Sci., 26, 991-996.

Warwick, J. W., 1967 : Radiophysics of Jupiter. Space Sci. Rev., 6, 841-891.

Westphal, J. A., 1969: Observations of localized 5-micron radiation from Jupiter. Astrophys. $J$, 157, 63. 